\author{
Zh.A. Balkizov \\ Institute of Applied Mathematics and Automation of Kabardin-Balkar \\ Scientific Centre of RAS, Nalchik, Russia \\ (E-mail: Giraslan@yandex.ru)
}

\title{
The first boundary value problem with deviation from the characteristics for a second-order parabolic-hyperbolic equation
}

We pose and investigate the first boundary value problem using a model second order equation of parabolic-hyperbolic type with A.M. Nakhushev's conditions violated relative to coefficients. Despite these conditions are violated an a priori estimate similar to the a priori estimate obtained by A.M. Nakhushev takes place for solving the first boundary-value problem under study.

Keywords: equation of mixed parabolic-hyperbolic type, the first boundary value problem, a priori estimate of the solution.

\section{Introduction}

Consider the equation

$$
f=L u \equiv \begin{cases}u_{y y}-k(y) u_{x x}, & y<0 \\ u_{y y}+u_{x}, & y>0\end{cases}
$$

where $k(y) \geq k_{1}>0, f=f(x, y)$ are given functions, and $u=u(x, y)$ is an unknown one. For $y<0$, equation (1) coincides with the Chaplygin equation [1;21], and for $y>0$, it is a parabolic equation backward in time (with $x$ standing for a time variable). Thus, equation (1) is a second-order parabolic-hyperbolic equation with non-characteristic line of degeneracy [2].

A great number of scientific researches are devoted to the study of boundary-value problems for secondorder parabolic-hyperbolic equations with non-characteristic line of degeneracy. For example, in [3], by the spectral method, a priori estimates in the $L_{p^{-}}$and $C$-classes for solution of the Tricomi problem for an equation of the form (1), are obtained. In [4-8], boundary value problems with deviation from the characteristics for parabolic-hyperbolic equations are studied.

In [9], a method enabling one to formulate well-posed boundary value problems for a class of linear parabolichyperbolic equations of the form

$$
L u \equiv u_{y y}-k(x, y) u_{x x}+a(x, y) u_{x}+b(x, y) u_{y}+c(x, y) u=f(x, y),
$$

in a bounded domain $\Omega$ with piecewise smooth boundary $\Sigma$, is given. It is assumed that the coefficients of (2) are continuous and satisfy the Nakhushev conditions, namely:

$$
\begin{array}{r}
k_{x}(x, y), a_{x}(x, y), c_{x}(x, y) \in C(\bar{\Omega}), \quad f(x, y) \in L_{2}(\Omega), \\
k(x, y) \geq 0, \quad \forall(x, y) \in \Omega, \\
a(x, y)>0, \quad 2 a(x, y)+k_{x}(x, y) \geq 0, \quad \forall(x, y) \in \Omega_{0},
\end{array}
$$

where $\Omega_{0}$ is the domain of parabolicity of (2). In particular, all parts of $\Sigma$ which should be free from boundary data, are specified. Moreover, an a priori estimate for such a problem for (2) is obtained. The latter implies the uniqueness of a regular solution and the existance of a weak one. In [10], this problem is called the first boundary value problem for parabolic-hyperbolic equations.

In what follows, by $\Omega$ we denote the union of $\Omega_{1}, \Omega_{2}$ and $J_{r}$, i.e. $\Omega=\Omega_{1} \cup \Omega_{2} \cup J_{r}$, where

$$
\begin{gathered}
\Omega_{1}=\{(x, y): 0<x<r, 0<y<\varphi(x)\} \\
\Omega_{2}=\left\{(x, y): 0<x \leq l, \gamma_{1}(x)<y<0\right\} \cup\left\{(x, y): l \leq x<r, \gamma_{2}(x)<y<0\right\}
\end{gathered}
$$


and

$$
J_{r}=\{(x, y): 0<x<r, y=0\}
$$

It is assumed that

$$
\varphi(x) \in C^{1}[0, r], \quad \gamma_{1}(x) \in C^{1}[0, l], \quad \gamma_{2}(x) \in C^{1}[l, r]
$$

and

$$
\varphi(x)>0, \quad \gamma_{1}^{\prime}(x)<0, \quad \gamma_{2}^{\prime}(x)>0, \quad \gamma_{1}(0)=\gamma_{2}(r)=0, \quad \gamma_{1}(l)=\gamma_{2}(l) .
$$

We will use the following notations:

$$
\begin{gathered}
A=A(0,0) ; \quad B=B(r, 0) ; \quad C=C\left(l, \gamma_{1}(l)\right) ; \quad A_{0}=A_{0}(0, \varphi(0)) ; \quad B_{0}=B_{0}(r, \varphi(r)) ; \\
\sigma_{0}=\{(x, y): 0<x<r, y=\varphi(x)\} ; \quad \sigma_{1}=\left\{(x, y): 0<x<l, y=\gamma_{1}(x)\right\}
\end{gathered}
$$

and

$$
\sigma_{2}=\left\{(x, y): l<x<r, y=\gamma_{2}(x)\right\} .
$$

We also require $f(x, y)$ and $k(y)$ to be continuous, i.e.

$$
f \in C\left(\bar{\Omega}_{i}\right), \quad i=1,2 ; \quad k(y) \in C\left[\gamma_{1}(l), 0\right] .
$$

As it is shown in [9], the well-posedness of the first boundary-value problem for equation (1) strongly depends on the mutual location of curves $\sigma_{1}$ and $\sigma_{2}$, and the characteristics

$$
A C_{1}: x+\int_{0}^{y} \sqrt{k(t)} d t=0 \quad \text { and } \quad C_{1} B: x-\int_{0}^{y} \sqrt{k(t)} d t=r .
$$

Here, $C_{1}$ is the point of intersection of the characteristics passing through the points $A$ and $B$.

In this paper, we consider equation (1) with coefficients not satisfying the Nakhushev conditions. We state and solve the first boundary value problem in the case when the curves $\sigma_{1}$ and $\sigma_{2}$ lie entirely in the characteristic triangle $A B C_{1}$. We also prove an analogue of the a priori estimate obtained in [9].

\section{Formulation of the problem}

By substitution [9]:

$$
u(x, y)=v(x, y) \exp (\mu x),
$$

the given operator $L u$ associated with the operator $L_{\mu} v$ according to the formula

$$
L_{\mu} v= \begin{cases}v_{y y}-k(y) v_{x x}-2 \mu k(y) v_{x}-\mu^{2} k(y) v, & y<0 \\ v_{y y}+v_{x}+\mu v, & y>0\end{cases}
$$

where $\mu$ is some negative number. The operators $L u$ and $L_{\mu} v$ when replacing (6) will be related by

$$
L u=\exp (\mu x) L_{\mu} v .
$$

The Regular solution of the equation

$$
L_{\mu} v=f_{\mu}, f_{\mu}=\exp (-\mu x) f
$$

in the domain $\Omega$ we call any function $v=v(x, y)$ for the class $C(\bar{\Omega}) \cap C^{1}(\Omega) \cap C^{2}\left(\Omega_{1}\right) \cap C_{y}^{2}\left(\Omega_{2}\right)$, which turns the equation into an identity upon substitution.

Problem 1. Find in the domain $\Omega$ the regular solution $v=v(x, y)$ to the equation (7) of $v(x, y) \in C^{1}(\bar{\Omega}) \cap C^{2}\left(\Omega_{i}\right), i=\overline{1,2}$ satisfying the boundary conditions

$$
\begin{gathered}
v=0 \quad \forall(x, y) \in B B_{0} \cup \sigma_{0} \cup \sigma_{2} ; \\
v_{y}=0 \quad \forall(x, y) \in \sigma_{1} .
\end{gathered}
$$


Theorem to get a priori estimation of the solution to problem 1

Further, we use the following notation:

$$
\begin{gathered}
(u, v)_{0}=\int_{\Omega} u v d \Omega ; \quad\|u\|_{0}^{2}=\int_{\Omega} u^{2} d \Omega \\
\|u\|_{1}^{2}=\int_{\Omega}\left[u^{2}+u_{x}^{2}+u_{y}^{2}\right] d \Omega .
\end{gathered}
$$

The following Theorem holds true.

Theorem 1. Assume the curves $\sigma_{0}, \sigma_{1}$ and $\sigma_{2}$, restricting the domain $\Omega$ are such that they possess the following properties:

$$
\begin{gathered}
\sigma_{0}: y=\varphi(x) \in C^{1}[0, r], \text { and besides } \varphi^{\prime}(x) \leq 0 \quad \forall x \in[0, r] ; \\
\sigma_{1}: y=\gamma_{1}(x) \in C^{1}[0, l] ; \sigma_{2}=C B: y=\gamma_{2}(x) \in C^{1}[l, r] ; \\
-1<\sqrt{k(y)} \gamma_{1}^{\prime}(x)<0 \quad \forall(x, y) \in A C ; \\
0<\sqrt{k(y)} \gamma_{2}^{\prime}(x) \leq 1 \quad \forall(x, y) \in C B .
\end{gathered}
$$

Then to solve $v=v(x, y)$ of problem 1 we have the energy inequality

$$
\|v\|_{1} \leq M_{1}\left\|f_{\mu}\right\|_{0},
$$

where the function $v=v(x, y)$ is associated with the solution $u=u(x, y)$ of original equation (1) according to the formula (6), and $M_{1}$ is the positive constant that does not depend on the sought function $v(x, y)$.

Proof. For the operator $L_{\mu} v$ as $y<0$ the equality

$$
\begin{gathered}
2 \delta(x) v_{x} L_{\mu} v=2 \delta(x) v_{x}\left[v_{y y}-k(y) v_{x x}-2 \mu k(y) v_{x}-\mu^{2} k(y) v\right]= \\
=\frac{\partial}{\partial y}\left[2 \delta(x) v_{x} v_{y}\right]-\frac{\partial}{\partial x}\left[\delta(x)\left(k(y) v_{x}^{2}+v_{y}^{2}+\mu^{2} k(y) v^{2}\right)\right]+ \\
+\delta(x)\left[\alpha\left(k(y) v_{x}^{2}+v_{y}^{2}\right)-4 \mu k(y) v_{x}^{2}+\alpha \mu^{2} k(y) v^{2}\right]
\end{gathered}
$$

holds true while as $y>0$ we have the equality

$$
\begin{gathered}
2 \delta(x) L_{\mu} v=2 \delta(x)\left[v_{y y}+v_{x}+\mu v\right]=\frac{\partial}{\partial x}\left[\delta(x)\left(\mu v^{2}-v_{y}^{2}\right)\right]+\frac{\partial}{\partial y}\left[2 \delta(x) v_{x} v_{y}\right]+ \\
+\delta(x)\left[2 v_{x}^{2}+\alpha v_{y}^{2}-\mu \alpha v^{2}\right]
\end{gathered}
$$

where $\delta(x)=\exp (\alpha x), \alpha>0$. With the above equalities, it is easy to verify that for any function $v(x, y) \in C^{1}(\bar{\Omega}) \cap C^{2}\left(\Omega_{i}\right), i=\overline{1,2}$ holds

$$
\begin{gathered}
2\left(\delta(x) v_{x}, L_{\mu} v\right)_{0}=\int_{\Omega} 2 \delta(x) v_{x} L_{\mu} v d \Omega=\int_{\Omega_{1}} 2 \delta(x) v_{x} L_{\mu} v d \Omega_{1}+\int_{\Omega_{2}} 2 \delta(x) v_{x} L_{\mu} v d \Omega_{2}= \\
=\int_{\Omega_{1}} 2 \delta(x) v_{x}\left[v_{y y}-k(y) v_{x x}-2 \mu k(y) v_{x}-\mu^{2} k(y) v\right] d \Omega_{1}+ \\
\quad+\int_{\Omega_{2}} 2 \delta(x) v_{x}\left[v_{y y}+v_{x}+\mu v\right] d \Omega_{2}= \\
=\int_{\Omega_{1}} \frac{\partial}{\partial y}\left[2 \delta(x) v_{x} v_{y}\right]-\frac{\partial}{\partial x}\left[\delta(x)\left(k(y) v_{x}^{2}+v_{y}^{2}+\mu^{2} k(y) v^{2}\right)\right] d \Omega_{1}+
\end{gathered}
$$




$$
\begin{gathered}
+\int_{\Omega_{2}} \frac{\partial}{\partial x}\left[\delta(x)\left(\mu v^{2}-v_{y}^{2}\right)\right]+\frac{\partial}{\partial y}\left[2 \delta(x) v_{x} v_{y}\right] d \Omega_{2}+ \\
+\int_{\Omega_{1}} \delta(x)\left[\alpha\left(k(y) v_{x}^{2}+v_{y}^{2}\right)-4 \mu k(y) v_{x}^{2}+\alpha \mu^{2} k(y) v^{2}\right] d \Omega_{1}+ \\
+\int_{\Omega_{2}} \delta(x)\left[2 v_{x}^{2}+\alpha v_{y}^{2}-\mu \alpha v^{2}\right] d \Omega_{2} .
\end{gathered}
$$

Applying the Green formula to the last equation, we obtain

$$
\begin{aligned}
& 2\left(\delta(x) v_{x}, L_{\mu} v\right)_{0}=-\int_{\Gamma_{1}}\left[2 \delta(x) v_{x} v_{y}\right] d x+\left[\delta(x)\left(k(y) v_{x}^{2}+v_{y}^{2}+\mu^{2} k(y) v^{2}\right)\right] d y+ \\
& +\int_{\Gamma_{2}}\left[\delta(x)\left(\mu v^{2}-v_{y}^{2}\right)\right] d y-\left[2 \delta(x) v_{x} v_{y}\right] d x+ \\
& +\int_{\Omega_{1}} \delta(x)\left[(\alpha-4 \mu) k(y) v_{x}^{2}+\alpha v_{y}^{2}+\alpha \mu^{2} k(y) v^{2}\right] d \Omega_{1}+ \\
& +\int_{\Omega_{2}} \delta(x)\left[2 v_{x}^{2}+\alpha v_{y}^{2}-\mu \alpha v^{2}\right] d \Omega_{2}= \\
& =\int_{A}^{A_{0}} \delta(x)\left[v_{y}^{2}(0, y)-\mu v^{2}(0, y)\right] d y+\int_{B}^{B_{0}} \delta(x)\left[\mu v^{2}(r, y)-v_{y}^{2}(r, y)\right] d y+ \\
& +\int_{A_{0}}^{B_{0}} \delta(x)\left[2 v_{x} v_{y} d x+\left(v_{y}^{2}-\mu v^{2}\right) d y\right]- \\
& -\int_{A}^{C} \delta(x)\left[2 v_{x} v_{y} d x+\left(k(y) v_{x}^{2}+v_{y}^{2}+\mu^{2} k(y) v^{2}\right) d y\right]- \\
& -\int_{C}^{B} \delta(x)\left[2 v_{x} v_{y} d x+\left(k(y) v_{x}^{2}+v_{y}^{2}+\mu^{2} k(y) v^{2}\right) d y\right]+ \\
& +\int_{\Omega_{1}} \delta(x)\left[(\alpha-4 \mu) k(y) v_{x}^{2}+\alpha v_{y}^{2}+\alpha \mu^{2} k(y) v^{2}\right] d \Omega_{1}+ \\
& +\int_{\Omega_{2}} \delta(x)\left[2 v_{x}^{2}+\alpha v_{y}^{2}-\mu \alpha v^{2}\right] d \Omega_{2}= \\
& =I_{1}+I_{2}+I_{3}+I_{4}+I_{5}+I_{6}+I_{7},
\end{aligned}
$$

where $\Gamma_{1}=A A_{0} \cup A_{0} B_{0} \cup B B_{0} \cup A B ; \Gamma_{2}=A C \cup C B \cup A B$ - are boundaries of the domains $\Omega_{1}$ and $\Omega_{2}$, respectively.

Since $\mu<0$ then

$$
I_{1}=\int_{A}^{A_{0}} \delta(x)\left[v_{y}^{2}(0, y)-\mu v^{2}(0, y)\right] d y \geq 0
$$


and due to boundary condition (8)

$$
I_{2}=\int_{B}^{B_{0}} \delta(x)\left[\mu v^{2}(r, y)-v_{y}^{2}(r, y)\right] d y=0
$$

Next, in view of boundary condition (8) $\left.v(x, y)\right|_{A_{0} B_{0}}=\left.v(x, y)\right|_{y=\varphi(x)}=0$, and therefore in the line $A_{0} B_{0}$ the equality: $v_{x}+v_{y} \varphi^{\prime}(x)=0$ holds. Thus,

$$
\begin{gathered}
I_{3}=\int_{A_{0}}^{B_{0}} \delta(x)\left[2 v_{x} v_{y} d x+\left(v_{y}^{2}-\mu v^{2}\right) d y\right]=\int_{0}^{r} \delta(x)\left[2 v_{x} v_{y}+v_{y}^{2} \varphi^{\prime}(x)\right] d x= \\
=\int_{0}^{r} \delta(x)\left[-2 \varphi^{\prime}(x) v_{y}^{2}+\varphi^{\prime}(x) v_{y}^{2}\right] d x=-\int_{0}^{r} \delta(x) \varphi^{\prime}(x) v_{y}^{2} d x
\end{gathered}
$$

and with condition (10) for the function $y=\varphi(x)$ the integral $I_{3} \geq 0$.

On the curve $\sigma_{1}: y=\gamma_{1}(x)$ taking into account the boundary condition (9) we have

$$
\begin{gathered}
I_{4}=-\int_{A}^{C} \delta(x)\left[2 v_{x} v_{y} d x+\left(k(y) v_{x}^{2}+v_{y}^{2}+\mu^{2} k(y) v^{2}\right) d y\right]= \\
=-\int_{0}^{l} \delta(x)\left[2 v_{x} v_{y}+\left(k(y) v_{x}^{2}+v_{y}^{2}+\mu^{2} k(y) v^{2}\right) \gamma_{1}^{\prime}(x)\right] d x= \\
=-k(y) \int_{0}^{l} \gamma_{1}^{\prime}(x) \delta(x)\left[v_{x}^{2}+\mu^{2} v^{2}\right] d x \geq 0 .
\end{gathered}
$$

Similarly on the curve $\sigma_{2}: y=\gamma_{2}(x)$ get

$$
\begin{gathered}
I_{5}=-\int_{C}^{B} \delta(x)\left[2 v_{x} v_{y} d x+\left(k(y) v_{x}^{2}+v_{y}^{2}+\mu^{2} k(y) v^{2}\right) d y\right]= \\
=-\int_{l}^{r} \delta(x)\left[2 v_{x} v_{y}+\left(k(y) v_{x}^{2}+v_{y}^{2}+\mu^{2} k(y) v^{2}\right) \gamma_{2}^{\prime}(x)\right] d x= \\
=\int_{l}^{r} \delta(x)\left[-k(y) \gamma_{2}^{\prime}(x) v_{x}^{2}-2 v_{x} v_{y}-\gamma_{2}^{\prime}(x) v_{y}^{2}-\mu^{2} k(y) \gamma_{2}^{\prime}(x) v^{2}\right] d x .
\end{gathered}
$$

Due to condition (8) of problem 1: $\left.v(x, \quad y)\right|_{\sigma_{2}}=\left.v(x, \quad y)\right|_{y=\gamma_{2}(x)}=0$. Hence $\left.\left[v_{x}(x, y)+v_{y}(x, y) \gamma_{2}^{\prime}(x)\right]\right|_{\sigma_{2}=C B}=0$. Considering this, for the integral $I_{5}$ we have

$$
I_{5}=\int_{l}^{r} \delta(x)\left[\gamma_{2}^{\prime}(x) v_{y}^{2}-k(y) \gamma_{2}^{\prime 3}(x) v_{y}^{2}\right] d x=\int_{l}^{r} \delta(x) \gamma_{2}^{\prime}(x)\left[1-k(y) \gamma_{2}^{\prime 2}(x)\right] v_{y}^{2} d x .
$$

By the last formula it is clear under conditions (11), (13) on the curve $\sigma_{2}=C B: y=\gamma_{2}(x)$ for integral $I_{5}$ we get the inequality

$$
I_{5}=\int_{l}^{r} \delta(x) \gamma_{2}^{\prime}(x)\left[1-k(y) \gamma_{2}^{\prime 2}(x)\right] v_{y}^{2} d x \geq 0
$$


Thus, under conditions (10)-(13) of theorem 1 the integrals $I_{n} \geq 0, n=\overline{1,5}$. Discarding the nonnegative integrals $I_{n}, n=\overline{1,5}$ by (15) we arrive at the inequality

$$
\begin{gathered}
2\left(\delta(x) v_{x}, L_{\mu} v\right)_{0} \geq \\
\geq \int_{\Omega_{1}} \delta(x)\left[(\alpha-4 \mu) k(y) v_{x}^{2}+\alpha v_{y}^{2}+\alpha \mu^{2} k(y) v^{2}\right] d \Omega_{1}+ \\
+\int_{\Omega_{2}} \delta(x)\left[2 v_{x}^{2}+\alpha v_{y}^{2}-\mu \alpha v^{2}\right] d \Omega_{2}= \\
=\int_{\Omega} \delta(x)\left[(2 H(y)+(\alpha-4 \mu) k(y) H(-y)) v_{x}^{2}+\alpha v_{y}^{2}\right] d \Omega+ \\
+\alpha \int_{\Omega} \delta(x)\left[\mu^{2} H(-y)-\mu H(y)\right] v^{2} d \Omega,
\end{gathered}
$$

where $H(y)$ is the Heaviside function.

On the other hand, with the Cauchy-Bunyakovskii inequality, for all $\varepsilon>0$ find

$$
\begin{gathered}
2\left(\delta(x) v_{x}, L_{\mu} v\right)_{0}=2\left(\sqrt{\varepsilon \delta(x)} v_{x}, \frac{\sqrt{\delta(x)} L_{\mu} v}{\sqrt{\varepsilon}}\right)_{0} \leq \\
\leq \varepsilon\left\|\sqrt{\delta(x)} v_{x}\right\|_{0}^{2}+C(\varepsilon)\left\|\sqrt{\delta(x)} L_{\mu} v\right\|_{0}^{2} .
\end{gathered}
$$

By (16) and (17) the inequality

is implied, whence

$$
\begin{aligned}
& \int_{\Omega} \delta(x)\left[(2 H(y)+(\alpha-4 \mu) k(y) H(-y)) v_{x}^{2}+\right. \\
& \left.\quad+\alpha v_{y}^{2}+\alpha\left(\mu^{2} H(-y)-\mu H(y)\right) v^{2}\right] d \Omega \leq \\
& \quad \leq \varepsilon\left\|\sqrt{\delta(x)} v_{x}\right\|_{0}^{2}+C(\varepsilon)\left\|\sqrt{\delta(x)} L_{\mu} v\right\|_{0}^{2}
\end{aligned}
$$

$$
\begin{gathered}
\int_{\Omega} \delta(x)\left[(2 H(y)+(\alpha-4 \mu) k(y) H(-y)-\varepsilon) v_{x}^{2}+\alpha v_{y}^{2}+\right. \\
\left.+\alpha\left(\mu^{2} H(-y)-\mu H(y)\right) v^{2}\right] d \Omega \leq C_{1}(\varepsilon)\left\|L_{\mu} v\right\|_{0}^{2},
\end{gathered}
$$

where $C_{1}(\varepsilon)=\exp (\alpha r) C(\varepsilon)$. Select in the latter inequality the numbers $\varepsilon>0, \alpha>0, \mu<0$ so that $\varepsilon<\min \left\{(\alpha-4 \mu) k_{1}, 2\right\}$. Then for the left-hand side the following estimate holds:

$$
\begin{gathered}
\int_{\Omega} \delta(x)\left[(2 H(y)+(\alpha-4 \mu) k(y) H(-y)-\varepsilon) v_{x}^{2}+\alpha v_{y}^{2}+\right. \\
\left.+\alpha\left(\mu^{2} H(-y)-\mu H(y)\right) v^{2}\right] d \Omega \geq M \int_{\Omega}\left(v^{2}+v_{x}^{2}+v_{y}^{2}\right) d \Omega=M\|v\|_{1}^{2},
\end{gathered}
$$

where $M=\min \left\{2-\varepsilon ;(\alpha-4 \mu) k_{1}-\varepsilon ; \alpha ; \mu^{2} \alpha,|\mu| \alpha\right\}$.

By inequalities (18)-(19) we arrive at the a priori estimate (14). Theorem 1 is proved.

By the Theorem 1 we conclude that if $u=u(x, y)$ in the domain $\Omega$ is the solution of original equation (1) for the class $C^{1}(\bar{\Omega})$ with the right-hand side $f(x, y) \in L_{2}(\Omega)$ satisfying the boundary conditions

$$
u=0 \quad \forall(x, y) \in B B_{0} \cup \sigma_{0} \cup \sigma_{2},
$$

then we have the estimate

$$
\int_{\Omega}\left[\left(u_{x}-\mu u\right)^{2}+u_{y}^{2}+u^{2}\right] d \Omega \leq M_{1}^{2}\|f\|_{0}^{2} .
$$


By the a priori estimate (20) implies the uniqueness of the regular solution of Problem 1 and the existence of a weak solution for the dual of Problem 1 for any right-hand side $f(x, y) \in L_{2}(\Omega)$.

We note that boundary condition (9) in the statement of Problem 1 can be replaced either by the condition $v_{x}=0 \quad \forall(x, y) \in \sigma_{1}$ or the condition $v_{n}=0 \quad \forall(x, y) \in \sigma_{1}$, where $v_{n}$ is the derivative of the function $v=v(x, y)$ in the direction of the outward-pointing normal to the curve $\sigma_{1}=A C$.

\section{References}

1 Чаплыгин С.А. О газовых струях / С.А. Чаплыгин. - М.-Л.: Гос. изд-во техн.-теор. лит., 1949. $144 \mathrm{c}$.

2 Сабитов К.Б. К теории уравнений смешанного параболо-гиперболического типа со спектральным параметром / К.Б. Сабитов // Дифференц. уравнения. - 1989. - Т. 25. - № 1. - С. 117-126.

3 Капустин Н.Ю. Задачи для параболо-гиперболических уравнений и соответствующие спектральные вопросы с параметром в граничных точках: дис. ... д-ра физ.-мат. наук: 01.01.01 - Математический анализ / Капустин Николай Юрьевич. - М.: Изд-во МГУ, 2012. - 172 с.

4 Елеев В.А. Аналог задачи Трикоми для смешанных параболо-гиперболических уравнений с нехарактеристической линией изменения типа / В.А. Елеев // Дифференц. уравнения. - 1977. - Т. 13. - № $1 .-$ C. $56-63$.

5 Бердышев А.С. Об одном аналоге нелокальной краевой задачи для параболо-гиперболического уравнения в области с отходом от характеристики / А.С. Бердышев, М.А. Садыбеков // Узбекский математический журнал. - 1991. - № 6. - С. 14-19.

6 Салахитдинов М.С. Краевые задачи для параболо-гиперболического уравнения в области с отходом от характеристики / М.С. Салахитдинов, А.С. Бердышев // Докл. РАН. - 1992. - Т. 327. - № 3. - C. 303-305.

7 Бердышев А.С. Краевые задачи с наклонной производной для параболо-гиперболического уравнения в области с отходом от характеристики / А.С. Бердышев, Г.Д. Тойжанова // Известия Национальной академии наук Республики Казахстан. Сер. Физико-математические науки. $-1995 .-$ № 5. - C. $13-20$.

8 Berdyshev A.S. On the Volterra property of a boundary problem with integral gluing condition for a mixed parabolic-hyperbolic equation / A.S. Berdyshev, A. Cabada, E.T. Karimov, N.S. Akhtaeva // Boundary Value Problems. - 2013. - 1(94).

9 Нахушев А.М. К теории линейных краевых задач для уравнения второго порядка смешанного гиперболо-параболического типа / А.М. Нахушев // Дифференц. уравнения. - 1978. - Т. 14. № 1. - C. $66-73$.

10 Нахушев А.М. Задачи со смещением для уравнений в частных производных / А.М. Нахушев. - М.: Наука, 2006. - 287 с.

Ж.А. Балкизов

\section{Гиперболану облысында сипаттаушылардан алыстаған екінші ретті парабола-гиперболалық типті моделді теңдеу үшін бірінші шеттік есеп}

Екінші ретті моделді парабола-гиперболалық типті теңдеу мысалында коэффициенттеріне қатысты А.М. Нахушевтің шарттары орындалмаған жағдайы үшін бірінші шеттік есеп қойылып зерттелген. Коэффициенттеріне қатысты А.М. Нахушевтің шарттары орындалмаған жағдайға қарамастан, жұмыста зерттелініп отырған бірінші шеттік есеп үшін А.М. Нахушевтің жұмыстарында алынған априорлық бағалауға ұқсас априорлық бағалаудың орын алатыны көрсетілген.

Kiлm сөздер: параболикалық-гиперболалық теңдеу, бірінші шекаралық есеп, проблеманы шешуге априорлы бағалау. 
Ж.А. Балкизов

\section{Первая краевая задача для модельного уравнения параболо-гиперболического типа второго порядка с отходом от характеристик в области гиперболичности}

На примере модельного уравнения параболо-гиперболического типа второго порядка, относительно коэффициентов которого нарушены условия А.М. Нахушева, сформулирована и исследована первая краевая задача. Показано, что несмотря на то, что относительно коэффициентов рассматриваемого уравнения нарушены условия А.М. Нахушева, для решения исследуемой в работе первой краевой задачи будет иметь место априорная оценка, аналогичная априорной оценке, полученной А.М. Нахушевым.

Ключевые слова: уравнение смешанного параболо-гиперболического типа, первая краевая задача, априорная оценка решения.

\section{References}

1 Chaplygin, S.A. (1949). O hazovykh struiakh [On gas jets]. Moscow-Leningrad: Hosudarstvennoe izdatelstvo tekhniko-teoreticheskoi literatury [in Russian].

2 Sabitov, K.B. (1989). K teorii uravnenii smeshannoho parabolo-hiperbolicheskoho tipa so spektralnym parametrom [On the theory of equations of mixed parabolic-hyperbolic type with a spectral parameter]. Differentsialnye uravneniia - Differential equations, Vol. 25, 1, 93-100 [in Russian].

3 Kapustin, N.Yu. (2012). Zadachi dlia parabolo-hiperbolicheskikh uravnenii i sootvetstvuiushchie spektralnye voprosy s parametrom $\mathrm{v}$ hranichnykh tochkakh [Boundary value problems for parabolic-hyperbolic equations and specified spectral issues with a parameter at boundary points]. Doctor's thesis. Moscow: MHU [in Russian].

4 Eleev, V.A. (1977). Analoh zadachi Trikomi dlia smeshannykh parabolo-hiperbolicheskikh uravnenii s nekharakteristicheskoi liniei izmeneniia tipa [An analogue of the Tricomi problem for mixed parabolichyperbolic equations with a noncharacteristic line of change of type]. Differentsialnye uravneniia Differential equations, Vol. 13, 1, 56-63 [in Russian].

5 Berdyshev, A.S., \& Sadybekov, M.A. (1991). Ob odnom analohe nelokalnoi kraevoi zadachi dlia parabolohiperbolicheskoho uravneniia $\mathrm{v}$ oblasti s otkhodom ot kharakteristiki [On an analogue of a nonlocal boundary-value problem for a parabolic-hyperbolic equation in a domain with a departure from the characteristic]. Uzbekskii matematicheskii zhurnal - The Uzbek Mathematical Journal, 6, 14-19 [in Russian].

6 Salakhitdinov, M.S., \& Berdyshev, A.S. (1992). Kraevye zadachi dlia parabolo-hiperbolicheskoho uravneniia $\mathrm{v}$ oblasti $\mathrm{s}$ otkhodom ot kharakteristiki [Boundary value problems for a parabolic-hyperbolic equation in a domain with departure from the characteristic. Doklady RAN - Reports of the Russian Academy of Sciences, Vol. 327, 3, 303-305 [in Russian].

7 Berdyshev, A.S., \& Toyzhanova, G.D. (1995). Kraevye zadachi s naklonnoi proizvodnoi dlia parabolohiperbolicheskoho uravneniia v oblasti s otkhodom ot kharakteristiki [Oblique derivative boundary-value problems for parabolic-hyperbolic equation in a domain with departure from the characteristic]. Izvestiia Natsionalnoi akademii nauk Respubliki Kazakhstan. Seriia fiziko-matematicheskie nauki-Izvestiya of the National Academy of Sciences of the Republic of Kazakhstan. Series of Phys.-Math. Science, No. 5, 13-20 [in Russian].

8 Berdyshev, A.S., Cabada, A., Karimov, E.T., \& Akhtaeva, N.S. (2013). On the Volterra property of a boundary problem with integral gluing condition for a mixed parabolic-hyperbolic equation. Boundary Value Problems, 1(94). 
9 Nakhushev, A.M. (1978). K teorii lineinykh kraevykh zadach dlia uravneniia vtoroho poriadka smeshannoho hiperbolo-parabolicheskoho tipa [On the theory of linear boundary value problems for a second order equation of mixed hyperbolic-parabolic type]. Differentsialnye uravneniia - Differential equations, Vol. 14, 1, 66-73 [in Russian].

10 Nakhushev, A.M. (2006). Zadachi so smeshcheniem dlia uravnenii v chastnykh proizvodnykh [Boundary value problems with shift for partial differential equations]. Moscow: Nauka [in Russian]. 